\title{
Perovskite/organic-semiconductor heterojunctions for ultrasensitive photodetection
}

\author{
Leilei Gu and Zhiyong Fan \\ Light: Science \& Applications (2017) 6, e17090; doi:10.1038/lsa.2017.90; published online 11 August 2017
}

Keywords: photodetectors; ultrahigh responsivity; perovskite; PEDOT:PSS; photogating effect

Broadband photodetectors with unprecedented responsivity were reported. It widens the application of organometal halide perovskites in highly sensitive, low-cost and flexible photodetectors. The fundamental device physics revealed will have significant impact on the design of future ultrasensitive photodetectors and other optoelectronic devices.

Photodetectors with ultrahigh responsivity are highly desirable for a broad spectrum of practical applications, such as image sensing, process control and safety monitoring. To date, however, it has remained a challenge to produce ultrasensitive photodetectors at a low cost because of high requirements regarding the material quality, and due to the limitations on the sophisticated fabrication process with conventional device structures. Recently, this field has been greatly inspired by the emergence of organometal halide perovskite materials, which have a structure of $\mathrm{ABX}_{3}\left(\mathrm{~A}=\right.$ organic molecules; $\mathrm{B}=\mathrm{Pb}^{2+}$ or $\mathrm{Sn}^{2+}$; and $\mathrm{X}=\mathrm{Cl}^{-}, \mathrm{I}^{-}$and/or $\mathrm{Br}^{-}$). These materials have demonstrated an ensemble of appealing material properties, including exceptional light absorption capabilities, wide-range tunable bandgaps and micrometer-scale long carrier diffusion lengths. These excellent properties can provide benefits for the performance of most optoelectronic devices, including photodetectors, solar cells and light-emitting diodes. For instance, researchers have conducted investigations on photodetectors based on $\mathrm{MAPbI}_{1-x} \mathrm{Br}_{x}$ nanowires, which exhibit responsivities as high as $1.2 \times 10^{4} \mathrm{AW}^{-1}$ to light wavelengths ranging from $400 \mathrm{~nm}$ to $780 \mathrm{~nm}^{1}$. The potency of perovskite materials to serve as excellent building blocks for ultrasensitive photodetectors has also been demonstrated. However, their responsivity is still relatively low compared to many reported ultrasensitive photodetectors ${ }^{2,3}$. Since the quality of currently available perovskite materials is already very high, it is challenging to further enhance the responsivity by simply improving the perovskite film quality. To this end, one particularly effective approach is to interface perovskite layers with other functional materials. For example, graphene/ $\mathrm{MAPbBr}_{2} \mathrm{I}$ hybrid structurebased photodetectors were reported to demonstrate an improved photoresponsivity of $6 \times 10^{5} \mathrm{AW}^{-1}{ }^{4}$. Within this hybrid structure, perovskite only serves as light-absorbing media, while graphene functions as the effective charge transport channel. However, graphene does not represent the best candidate for a photodetector, as its high conductivity will result in a high dark current, which will then lead to a low on/off ratio and specific detectivity. Meanwhile, a perovskite/ $\mathrm{MoS}_{2}$ device structure has been demonstrated to possess a photoresponsivity of $1.94 \times 10^{6} \mathrm{AW}^{-1}$. Although the performance is appreciable, a band structure mismatch still exists between $\mathrm{MoS}_{2}$ and $\mathrm{MAPbI}_{3}$. Therefore, additional efforts must be conducted to search for appropriate materials with which to improve the performance of perovskite photodetectors.

Recently, Yan and colleagues made significant progress in this regard. In their work, they present the first report of low-voltage, highgain photodetectors based on perovskite/organic-semiconductor vertical heterojunctions, which demonstrate a high responsivity of $\sim 10^{9}$ $\mathrm{AW}^{-1}$ and a specific detectivity of $\sim 10^{14}$ Jones in a broadband spectrum ranging from ultraviolet to near-infrared ${ }^{6}$. These figures of merit are unprecedented for perovskite material-based photodetectors and are orders of magnitude higher than those of state-of-the-art commercial Si photodetectors whose peak responsivities are usually lower than $1 \mathrm{AW}^{-1}$. Figure 1 illustrates the device structure and working mechanism of Yan's ultrasensitive photodetectors. Generally, the high performance of this device is primarily ascribed to a pronounced photogating effect, which arises from the proper band alignment of perovskite/organic layers and is further enhanced by the long carrier lifetime and strong light absorption characteristics of perovskite materials. In particular, in order to fabricate the device, $\mathrm{Cr} / \mathrm{Au}$ electrodes are patterned through photolithography, followed by the spin-coating of PEDOT:PSS and $\mathrm{MAPbI}_{3-x} \mathrm{Cl}_{x}$ layers. Thereafter, a vertical type II heterojunction is formed between the PEDOT:PSS and perovskite layers. Under illumination, a large number of photocarriers are generated in the perovskite layer, whereby the holes migrate from the perovskite layer to the PEDOT:PSS layer driven by the aforementioned type II heterojunction, leaving the electrons within the perovskite layer. The efficient separation of photocarriers can dramatically prolong the carrier lifetime, which allows the holes to recirculate multiple times within the PEDOT:PSS channel, thereby resulting in a 


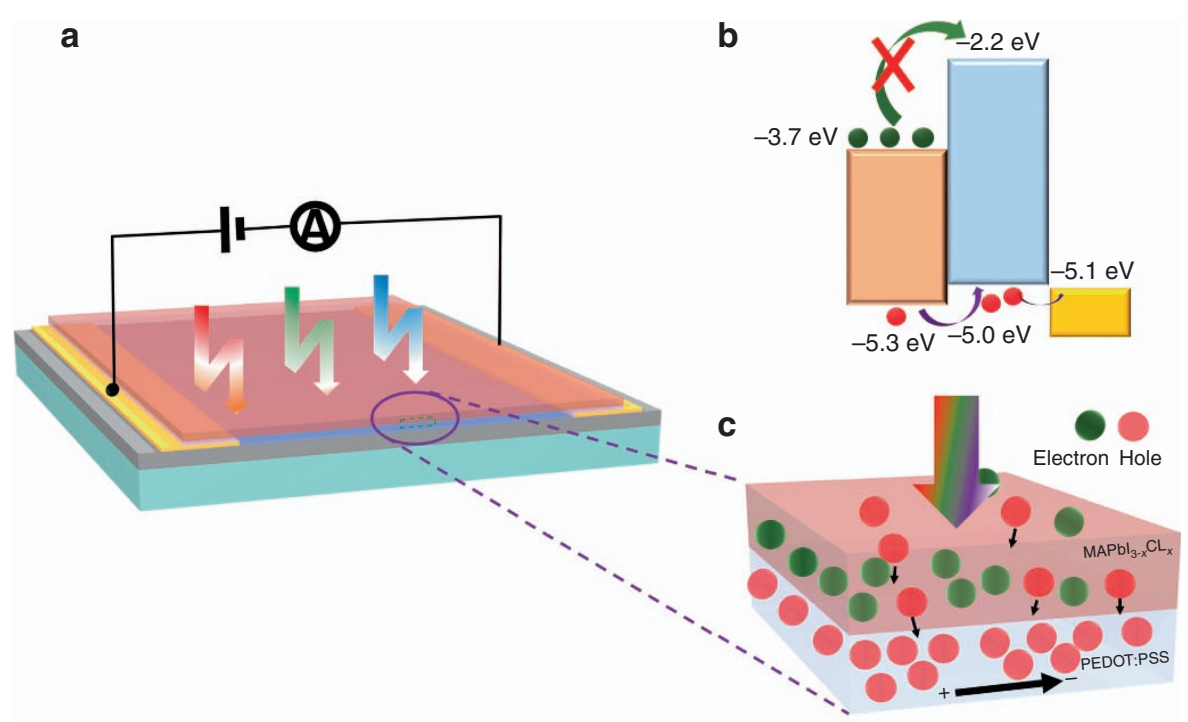

Figure 1 Device schematic (a), band alignment (b) and operation mechanism (c) of Yan and colleagues' ultrasensitive perovskite/organic-semiconductor heterojunction photodetectors.

high photoconductive gain and responsivity. In addition, the accumulated electrons inside the perovskite layer can function as a negatively biased gate electrode, which can further prolong the carrier lifetime and enhance the hole concentration in the PEDOT:PSS layer, giving rise to even higher photoconductive gains and responsivities. Yan and colleagues have revealed that the ultrahigh responsivity and gain of their photodetectors can be summarized by four explanations: (1) the long hole lifetime and diffusion length of $\mathrm{MAPbI}_{3-x} \mathrm{Cl}_{x}$ film can reduce recombination within the perovskite layer and consequently enhance the migration of holes from the perovskite layer from the $\mathrm{MAPbI}_{3-x} \mathrm{Cl}_{x}$ to the PEDOT:PSS layer; (2) the band alignment of these two materials allows for efficient carrier separation; (3) the high dielectric constant of $\mathrm{MAPbI}_{3-x} \mathrm{Cl}_{x}$ allows for additional charge accumulation within the perovskite layer under the same band bending conditions; (4) the high hole mobility in the PEDOT:PSS layer ensures fast hole transportation, and thus enhances the efficiency of carrier collection. These descriptions of device physics are instrumental and can serve as guidelines for the selection of appropriate materials for the future design of ultrasensitive photodetectors. In addition to the high performance of the photodetectors, the low temperature fabrication process makes it compatible with flexible substrates. Yan and colleagues have demonstrated flexible photodetectors exhibiting a high responsivity of $8.9 \times 10^{8} \mathrm{AW}^{-1}$, the performance of which can be maintained after 300 bending cycles, indicating a potential application for wearable and flexible electronics.

The work conducted by Yan and colleagues has demonstrated that broadband photodetectors based on a $\mathrm{MAPbI}_{3-x} \mathrm{Cl}_{x} / \mathrm{PEDOT}$ :PSS vertical heterojunction will demonstrate record-high values of the photoconductive gain and responsivity. This opens up future possibilities for applications of organometal halide perovskites within highly sensitive, low-cost and flexible photodetectors. More importantly, the fundamental physics revealed in this work can inspire the design of future high-performance photodetectors and other optoelectronic devices, including solar cells and light-emitting diodes.

\section{CONFLICT OF INTEREST}

The authors declare no conflict of interest.

1 Deng W, Huang LM, Xu XZ, Zhang XJ, Jin XC et al. Ultrahigh-responsivity photodetectors from perovskite nanowire arrays for sequentially tunable spectral measurement. Nano Lett 2017; 17: 2482-2489.

2 Liu X, Gu LL, Zhang QP, Wu JY, Long YZ et al. All-printable band-edge modulated ZnO nanowire photodetectors with ultra-high detectivity. Nat Commun 2014; 5: 4007.

3 Li LD, Gu LL, Lou Z, Fan ZY, Shen GZ. ZnO quantum dot decorated $\mathrm{Zn}_{2} \mathrm{SnO}_{4}$ nanowire heterojunction photodetectors with drastic performance enhancement and flexible ultraviolet image sensors. ACS Nano 2017; 11: 4067-4076.

4 Wang YS, Zhang YP, Lu Y, Xu WD, Mu HR et al. Hybrid graphene-perovskite phototransistors with ultrahigh responsivity and gain. Adv Opt Mater 2015; 3: 1389-1396.

5 Kang DH, Pae SR, Shim J, Yoo G, Jeon J et al. An ultrahigh-performance photodetector based on a perovskite-transition-metal-dichalcogenide hybrid structure. Adv Mater 2016; 28: 7799-7806.

6 Xie C, You P, Liu ZK, Li L, Yan F. Ultrasensitive broadband phototransistors based on perovskite/organic-semiconductor vertical heterojunctions. Light Sci Appl 2017; 6: e17023.

(c) (1) (2) This work is licensed under a Creative Commons Attributionc. other third party material in this article are included in the article's Creative Commons license, unless indicated otherwise in the credit line; if the material is not included under the Creative Commons license, users will need to obtain permission from the license holder to reproduce the material. To view a copy of this license, visit http:// creativecommons.org/licenses/by-nc-sa/4.0/

(C) The Author(s) 2017 